\title{
Population Variance Harmony Search Algorithm to Solve Optimal Power Flow with Non-Smooth Cost Function
}

\author{
B.K. Panigrahi, V. Ravikumar Pandi, Swagatam Das, and Ajith Abraham
}

\begin{abstract}
This chapter presents a novel Harmony Search (HS) algorithm used to solve security constrained optimal power flow (OPF) with various generator fuel cost characteristics. HS is a recently developed derivative-free, meta-heuristic optimization algorithm, which draws inspiration from the musical process of searching for a perfect state of harmony. This chapter analyses the evolution of the populationvariance over successive generations in HS and thereby draws some important attention regarding the explorative power of HS. This novel methodology of modified population variance harmony search algorithm (PVHS) easily takes care of solving optimal power flow problem even with non-smooth and piecewise cost functions. This PVHS algorithm was tested on the IEEE30 bus system with three different types of cost characteristics and compared with other reported results.
\end{abstract}

\section{Introduction}

Optimal power flow is the main tool used for planning an economic operation of power system [1]. In the recent attention in OPF shows the importance of the electric utilities to find the optimal secure operating point corresponding to the each loading condition. The problem of solving OPF involves estimating the optimal solution of control variables like generator real power, generator voltage magnitude and transformer tap settings corresponding to the best objective function. The

B.K. Panigrahi and V. Ravikumar Pandi

Department of Electrical Engineering, Indian Institute of Technology, Delhi,

Hauz Khas, New Delhi, India - 110016

E-mail: bkpanigrahi@ee.iitd.ac.in, ravikumarpandi@gmail.com

Swagatam Das

Department of Electronics and Telecommunication Engineering, Jadavpur University,

Kolkata - 700 032, India

E-mail: swagatamdas19@yahoo.co.in

Ajith Abraham

Machine Intelligence Research Labs (MIR Labs), USA

E-mail: ajith.abraham@ieee.org

Z.W. Geem: Recent Advances in Harmony Search Algorithm, SCI 270, pp. $65-75$. springerlink.com

(C) Springer-Verlag Berlin Heidelberg 2010 
dependent variable includes load bus voltage magnitude, generator reactive power generation, transmission line thermal loading. In general OPF is large scale highly non linear and constrained problem of minimizing the fuel cost.

OPF problem has been solved using many traditional techniques such as non linear programming, quadratic programming, mixed integer programming and interior point method. The literature review on these methods is given in Momoh et al. $[2,3]$. The disadvantage of these traditional methods is it cannot be applicable in case of the prohibited operating regions and multiple fuels. It also has higher sensitivity to initial solution, so it may trap into local optima. The difficulties in implementing OPF can be overcome by modern stochastic algorithms such as evolutionary programming (EP) [4], tabu search (TS) [5], improved evolutionary programming (IEP) [6], modified differential evolution (MDE) [7], particle swarm optimization (PSO) [9], genetic algorithm (GA) [10] and simulated annealing (SA) [11].

In 2001, Geem et al. proposed Harmony Search (HS) [13], a derivative-free, meta-heuristic algorithm, mimicking the improvisation process of music players. Since its inception, HS has found several applications in a wide variety of practical optimization problems like pipe-network design [14], structural optimization [15], vehicle routing problem [16], water distribution networks [17, 23], combined heat and power economic dispatch problem [18], Dam Scheduling [19] and numerical optimization [20]. The applicability of harmony search algorithm for discrete variable problem is given in [22]. The hybrid version of harmony search with particle swarm optimization applied to water network design is proposed in [24]. In the PVHS [21], the control parameter known as distance bandwidth (bw) has been made equal to the standard deviation of the current population. In this chapter we have used this PVHS algorithm to solve optimal power flow problem having various cost characteristics. The algorithm is applied to IEEE 30 bus test system effectively to show the appropriateness of the method. The simulation results with three different cost characteristics are comparable with the recently reported results.

\section{OPF Problem Formulation}

The objective of OPF problem is to minimize the total fuel cost of generators while satisfying several power system steady state security constraints. If $\mathrm{x}$ is the vector of state variables consisting of slack bus real power $\mathrm{P}_{g l}$, load bus voltages $\mathrm{V}_{\mathrm{L} k}$, generator reactive power outputs $\mathrm{Q}_{g j}$, and transmission line thermal loading $\mathrm{S}_{l}$, $\mathrm{x}$ can be expressed as:

$$
\mathrm{x}^{\mathrm{T}}=\left[\mathrm{P}_{g l}, \mathrm{~V}_{l l}, \ldots, \mathrm{V}_{l N L}, \mathrm{Q}_{g l}, \ldots, \mathrm{Q}_{g N G}, \mathrm{~S}_{l l}, \ldots, \mathrm{S}_{l N B}\right]
$$

where NL, NG and NB are the number of load buses, the number of generators and the number of transmission lines, respectively. $u$ is the vector of control variables consisting of real power outputs $\mathrm{P}_{g}$ except at the slack bus, generator voltages $\mathrm{V}_{\mathrm{g}}$, transformer tap settings $\mathrm{T}$. Hence, $u$ can be expressed as: 


$$
\mathrm{u}^{\mathrm{T}}=\left[\mathrm{P}_{g 2}, \ldots, \mathrm{P}_{g N G}, \mathrm{~V}_{g l}, \ldots, \mathrm{V}_{g N G}, \mathrm{~T}_{l}, \ldots, \mathrm{T}_{N T}\right]
$$

where NT is the number of regulating transformers.

The objective of OPF problem can be expressed as

$$
\text { Minimize } F=\sum_{j=1}^{N G} F_{i}\left(P_{g i}\right)
$$

where $F$ is the total generator fuel cost and $F_{i}$ is the fuel cost of generator connected to $\mathrm{i}^{\text {th }}$ bus. The system equality constraints $g(\mathrm{x}, \mathrm{u})$ is described by the following power balance equation

$$
\begin{gathered}
P_{g i}-P_{d i}=\sum_{j=1}^{N}\left|V_{i}\right|\left|V_{j} \| Y_{i j}\right| \cos \left(\theta_{i j}-\delta_{i}+\delta_{j}\right) \quad i=1, \ldots, N \\
Q_{g i}-Q_{d i}=-\sum_{j=1}^{N}\left|V_{i}\right|\left|V_{j} \| Y_{i j}\right| \sin \left(\theta_{i j}-\delta_{i}+\delta_{j}\right) \quad i=1, \ldots, N
\end{gathered}
$$

where $P_{g i}$ is the total real power generation at $\mathrm{i}^{\text {th }}$ bus, $P_{d i}$ is the total real power demand at $i^{\text {th }}$ bus, $Q_{g i}$ is the total reactive power generation at $i^{\text {th }}$ bus, $Q_{d i}$ is the total reactive power demand at $i^{\text {th }}$ bus, $\left|V_{i}\right|$ is the voltage magnitude at $i^{\text {th }}$ bus, $\left|V_{j}\right|$ is the voltage magnitude at $j^{\text {th }}$ bus, $\left|Y_{i j}\right|$ is the magnitude of the $i j^{\text {th }}$ element of Ybus, $\theta_{i j}$ angle of the $i j^{\text {th }}$ element of Ybus, $\delta_{i}$ voltage angle at $i^{\text {th }}$ bus and $\delta_{j}$ is the voltage angle at $j^{\text {th }}$ bus.

The system inequality constraints $h(\mathrm{x}, \mathrm{u})$ is consist of the following

1. Generator constraints: The generator real power outputs, reactive power outputs and voltages are bounded to its lower and upper limit.

$$
\begin{gathered}
P_{g i}^{\min } \leq P_{g i} \leq P_{g i}^{\max } \quad i=1, \ldots, N G \\
Q_{g i}^{\min } \leq Q_{g i} \leq Q_{g i}^{\max } \quad i=1, \ldots, N G \\
\left|V_{g i}^{\min }\right| \leq\left|V_{g i}\right| \leq\left|V_{g i}^{\max }\right| i=1, \ldots, N G
\end{gathered}
$$

where $P_{g i}^{\min }$ and $P_{g i}^{\max }$ are the minimum and maximum real power generation at $i^{\text {th }}$ generator bus, $Q_{g i}^{\min }$ and $Q_{g i}^{\max }$ are the minimum and maximum reactive power generation at $i^{\text {th }}$ generator bus, $\left|V_{g i}^{\min }\right|$ and $\left|V_{g i}^{\max }\right|$ are the minimum and maximum voltage magnitude at $i^{\text {th }}$ generator bus.

2. Transformer constraints: Transformer tap settings are bounded by minimum and maximum limits as follows

$$
T_{i}^{\min } \leq T_{i} \leq T_{i}^{\max } i=1, \ldots, N T
$$


where $T_{i}^{\min }$ and $T_{i}^{\max }$ are the minimum and maximum tap setting limit of $i^{\text {th }}$ transformer.

3. Security constraints: It includes the limits in the voltage magnitude of load buses and thermal loading limits of all transmission lines as follows.

$$
\begin{gathered}
\left|V_{L i}^{\min }\right| \leq\left|V_{L i}\right| \leq\left|V_{L i}^{\max }\right| i=1, \ldots, N L \\
\left|S_{l i}\right| \leq S_{l i}^{\max } \quad i=1, \ldots, N B
\end{gathered}
$$

where $\left|V_{L i}^{\min }\right|$ and $\left|V_{L i}^{\max }\right|$ are the minimum and maximum voltage magnitude at $i^{\text {th }}$ load bus, $S_{l i}^{\max }$ is the thermal limit of $i^{\text {th }}$ transmission line.

Constraints Handling: The problem of handling these constraints in the state variables are accommodated in the algorithm by including the constraints violation as quadratic penalty terms in the objective function itself.

$$
\begin{array}{r}
F_{\text {corr }}=F+K_{p}\left(P_{g 1}-P_{g 1}^{\lim }\right)^{2}+K_{v}\left(V_{L i}-V_{L i}^{\lim }\right)^{2}+ \\
K_{Q}\left(Q_{g i}-Q_{g i}^{\lim }\right)^{2}+K_{S}\left(\left|S_{l i}\right|-S_{l i}^{\lim }\right)^{2}
\end{array}
$$

where $\mathrm{K}_{\mathrm{p}}, \mathrm{K}_{\mathrm{v}}, \mathrm{K}_{\mathrm{Q}}$ and $\mathrm{K}_{\mathrm{S}}$ are the penalty factors corresponding to slack bus real power generation, load bus voltage magnitude, generator reactive power and transmission line thermal loadings, respectively. In the equation (12) the $x^{\lim }$ is equals to $\mathrm{x}_{\min }$ if $\mathrm{x}$ is lesser than the minimum limit and $\mathrm{x}_{\max }$ if $\mathrm{x}$ is greater than maximum limit.

\section{Harmony Search algorithm}

\subsection{Classical Harmony Search Algorithm}

In the harmony search algorithm musician improvises the pitches of his/her instrument to obtain a better state of harmony. The different steps of the classical HS algorithm are described below:

Step 1: The $1^{\text {st }}$ step is to specify the problem and initialize the parameter values. The optimization problem is defined as minimize (or maximize) $f(\mathbf{x})$ such that $x_{\min }^{i} \leq x_{i} \leq x_{\max }^{i}$, where $f(\mathbf{x})$ is the objective function, $\mathbf{x}$ is a solution vector consisting of $N$ decision variables $\left(x_{i}\right)$ and $x_{\min }^{i}$ and $x_{\max }^{i}$ are the lower and upper bounds of each decision variable, respectively. Other algorithm parameters, such as harmony memory size $(H M S)$, or the number of solution vectors in the harmony memory; harmony memory considering rate $(H M C R)$; pitch adjusting rate $(P A R)$; and the number of improvisations $(N I)$ or stopping criterion are also specified in this step. 
Step 2: The $2^{\text {nd }}$ step is to initialize the Harmony Memory. The initial harmony memory is generated from a uniform distribution in the ranges $\left[x_{\min }^{i}, x_{\max }^{i}\right]$, as

$$
x_{i}^{j}=x_{\min }^{i}+r \times\left(x_{\max }^{i}-x_{\min }^{i}\right)
$$

where $i=1,2, . ., N, j=1,2,3 \ldots ., H M S$, and $r \sim \mathrm{U}(0,1)$.

Step 3: The third step is known as the 'improvisation' step. The New Harmony vector $y=\left(y_{1}, y_{2}, \ldots, y_{N}\right)$ is generated by using memory consideration, pitch adjustment, and random selection. The procedure works as follows:

\section{Pseudo-code of improvisation in HS}

for each $i \in[1, N]$ do

if $U(0,1) \leq H M C R$ then /*memory consideration*/

$y_{i}=x_{i}^{j}$, where $j \sim U(1,2, \ldots, H M S)$.

if $U(0,1) \leq P A R$ then $/ *$ Pitch adjustment */

$$
Y_{i}=Y_{i}+r \times b w, \text { where } r \sim U(0,1)
$$

else $/ *$ random selection $* /$

$$
y_{i}=x_{\min }^{i}+r \cdot\left(x_{\max }^{i}-x_{\min }^{i}\right)
$$

endif

done

Step 4: In this step the harmony memory is updated. The generated harmony vector $y=\left(y_{1}, y_{2}, \ldots, y_{N}\right)$ replaces the worst harmony in the HM (harmony memory) only if its fitness is better than the worst harmony.

Step 5: The stopping criterion (generally the number of iterations) is checked. If it is satisfied, computation is terminated. Otherwise, Steps 3 and 4 are repeated.

\subsection{Modified Population Variance Harmony Search (PVHS) Algorithm}

In [21] Mukhopadhyay et al. analyze the explorative power in HS as follows:

Theorem 1. Let $x=\left\{x_{1}, x_{2}, \ldots, x_{N}\right\}$ be the current population, $Y=\left\{Y_{1}, Y_{2}, \ldots, Y_{N}\right\}$ the intermediate population obtained after harmony memory consideration and pitch adjustment. If HMCR be the harmony memory consideration probability, PAR the pitch-adjustment probability, bw the arbitrary distance bandwidth and if we consider the allowable range for the decision variables $\left(x_{i}\right)$ to be $\left\{x_{\min }, x_{\max }\right\}$ where $x_{\max }=a, x_{\min }=-a$, then 


$$
\begin{aligned}
\mathrm{E}(\operatorname{var}(Y))= & \frac{(m-1)}{m} \cdot\left[H M C R \cdot \operatorname{var}(x)+H M C R \cdot(1-H M C R) \cdot \bar{x}^{2}\right. \\
& +H M C R \cdot(1-H M C R) \cdot P A R \cdot b w \cdot \bar{x} \\
& \left.+H M C R \cdot P A R \cdot b w^{2} \cdot\left(\frac{1}{3}-\frac{H M C R \cdot P A R}{4}\right)+\frac{a^{2}}{3} \cdot(1-H M C R)\right]
\end{aligned}
$$

If $H M C R$ is chosen to be very high (i.e. very near to 1) and the distance bandwidth parameter $(b w)$ is chosen to be the standard deviation of the current population, then population variance (without selection) will grow almost exponentially over generations. Now, Neglecting the terms containing $(1-H M C R)$, and choosing $b w=\sigma(x)=\sqrt{\operatorname{var}(x)}$ the expression (16) becomes:

$$
\mathrm{E}(\operatorname{var}(Y))=\frac{(m-1)}{m} \cdot\left[H M C R+H M C R \cdot P A R \cdot\left(\frac{1}{3}-\frac{H M C R \cdot P A R}{4}\right)\right] \cdot \operatorname{var}(x)
$$

From equation (17) it is seen that if we do not include selection in the algorithm, then the expected variance of the $g^{\text {th }}$ population $\left(X_{g}\right)$ becomes:

$$
\mathrm{E}\left(\operatorname{var}\left(X_{g}\right)\right)=\left\{\frac{(m-1)}{m} \cdot\left[H M C R+H M C R \cdot P A R \cdot\left(\frac{1}{3}-\frac{H M C R \cdot P A R}{4}\right)\right]\right\}^{g} \cdot \operatorname{var}\left(X_{0}\right)
$$

In equation (10) if we choose the values of the parameters $H M C R, P A R$ in such a way that the term within the second brackets becomes greater than unity, then we can expect an exponential growth of population variance. This growth of expected population variance over generations gives the algorithm a strong explorative power. In modified HS the $b w$ is changed dynamically as

$$
\sigma(\mathrm{x})=\sqrt{\operatorname{var}(\mathrm{x})}
$$

We also took $H M C R=0.98$ and $P A R=0.67$ to equip the algorithm with more explorative power after performing a series of experiments.

\section{Implementation for OPF}

The optimal power flow problem is implemented using the PVHS algorithm by taking the control variables $u$ in the each harmony memory and equation (12) as objective. The algorithm stops when the current generation exceeds the total number of generation. The parameters are selected as: total number of generation = 100 , harmony memory size $(\mathrm{HMS})=50$, harmony memory considering rate $(\mathrm{HMCR})=0.98$, pitch adjusting rate $(\mathrm{PAR})=0.67$. The detailed implementation methodology is described as follows:

Step 1: Initialize harmony memory size (HMS), harmony memory considering rate (HMCR), pitch adjusting rate (PAR)

Step 2: Initialize harmonic memory and evaluate objective function after running load flow 
Step 3: Improvisation of harmony memory by pitch adjustment

Step 4: Run load flow and evaluate the objective function

Step 5: Update the harmony memory with this improvised solution if it is better than worst solution in memory

Step 6: If stopping criteria is met then print the OPF result and stop, otherwise go to step3.

\section{Results and Discussion}

The PVHS algorithm was tested on IEEE30 bus system consists of 6 generating units, 41 transmission lines and 4 tap-changing transformers [8]. The lower and upper limits on independent variables are shown in Table 1. In all the cases bus 1 is considered as swing bus. The simulation was done by taking a quadratic cost curve in case 1 , a piecewise quadratic cost curve in case 2 , and quadratic cost curve with valve point loading in case 3 . The result of the PVHS algorithm is compared with NLP [8], EP [4], TS [5], PSO [9], IEP [6] and MDE [7]. The algorithm is coded on Intel Pentium IV $2.3 \mathrm{GHz}$ processor and 2 GB RAM memory using Matlab 7.4 [12] programming language.

Table 1 Cost Results of PVHS Algorithm

\begin{tabular}{cccccc}
\hline Parameter & $\begin{array}{c}\text { Lower } \\
\text { Limit }\end{array}$ & $\begin{array}{c}\text { Upper } \\
\text { Limit }\end{array}$ & Case1 & Case2 & Case3 \\
\hline $\mathrm{P}_{\mathrm{g} 1}$ & 50 & 200 & 176.1824 & 139.9997 & 197.5413 \\
$\mathrm{P}_{\mathrm{g} 2}$ & 20 & 80 & 48.8268 & 54.9998 & 52.0571 \\
$\mathrm{P}_{\mathrm{g} 5}$ & 15 & 50 & 21.5131 & 24.0997 & 15.0000 \\
$\mathrm{P}_{\mathrm{g} 8}$ & 10 & 35 & 22.126 & 34.9994 & 10.0000 \\
$\mathrm{P}_{\mathrm{g} 11}$ & 10 & 30 & 12.224 & 18.4566 & 10.0000 \\
$\mathrm{P}_{\mathrm{g} 13}$ & 12 & 40 & 12.0011 & 17.9266 & 12.0000 \\
$\mathrm{~V}_{\mathrm{g} 1}$ & 0.95 & 1.05 & 1.0500 & 1.0500 & 1.0333 \\
$\mathrm{~V}_{\mathrm{g} 2}$ & 0.95 & 1.10 & 1.0381 & 1.0403 & 1.0100 \\
$\mathrm{~V}_{\mathrm{g} 5}$ & 0.95 & 1.10 & 1.0114 & 1.0145 & 0.9657 \\
$\mathrm{~V}_{\mathrm{g} 8}$ & 0.95 & 1.10 & 1.0192 & 1.0256 & 1.0307 \\
$\mathrm{~V}_{\mathrm{g} 11}$ & 0.95 & 1.10 & 1.0866 & 1.0786 & 1.0981 \\
$\mathrm{~V}_{\mathrm{g} 13}$ & 0.95 & 1.10 & 1.0847 & 1.0837 & 1.1000 \\
$\mathrm{~T}_{11}$ & 0.90 & 1.10 & 1.0247 & 1.0059 & 1.0978 \\
$\mathrm{~T}_{12}$ & 0.90 & 1.10 & 0.9267 & 0.9482 & 1.0983 \\
$\mathrm{~T}_{15}$ & 0.90 & 1.10 & 0.9993 & 1.0033 & 1.0703 \\
$\mathrm{~T}_{36}$ & 0.90 & 1.10 & 0.9422 & 0.9463 & 1.0141 \\
\hline $\mathrm{P}_{\text {loss }}$ & & & 9.4734 & 7.0818 & 13.1984 \\
Sum( $\left.\mathrm{P}_{\mathrm{g}}\right)$ & & & 292.8734 & 290.4818 & 296.5984 \\
Penalty & & & 0.0000 & 0.0000 & 0.0004 \\
Best Cost & & & $\mathbf{8 0 2 . 3 7 6 4}$ & $\mathbf{6 4 7 . 8 1 2 6}$ & $\mathbf{9 3 0 . 7 2 3}$ \\
\hline
\end{tabular}




\subsection{Case 1}

In this case the fuel cost characteristics of all the 6 generating units are given by quadratic cost function as

$$
f=\sum_{i=1}^{N G} F_{i}\left(P_{g i}\right)=\sum_{i=1}^{N G} a_{i}+b_{i} P_{g i}+c_{i} P_{g i}^{2}
$$

where $a_{i}, b_{i}$ and $c_{i}$ are the cost coefficients of the $i^{\text {th }}$ generator. The generator cost coefficients are found in [6] and the optimized parameters corresponding to minimum cost is given in Table 1 . The results of the PVHS algorithm is compared in Table 2 with other reported results. The statistical results of 50 trials are also reported in Table 2. The algorithm converges quickly and the results are better than others. The convergence characteristic of PVHS algorithm for this case is shown in Figure 1.

Table 2 Cost Comparison with Other Methods for Case 1

\begin{tabular}{cccccc}
\hline Parameter & NLP[8] & EP[4] & IEP[6] & MDE [7] & PVHS \\
\hline Best Cost & 802.40 & 802.62 & 802.465 & 802.376 & 802.3764 \\
Worst cost & - & 805.61 & 802.581 & 802.404 & 802.3912 \\
Avg cost & - & 803.51 & 802.521 & 802.382 & 802.3805 \\
Std cost & - & - & 0.039 & - & 0.0135 \\
\hline
\end{tabular}

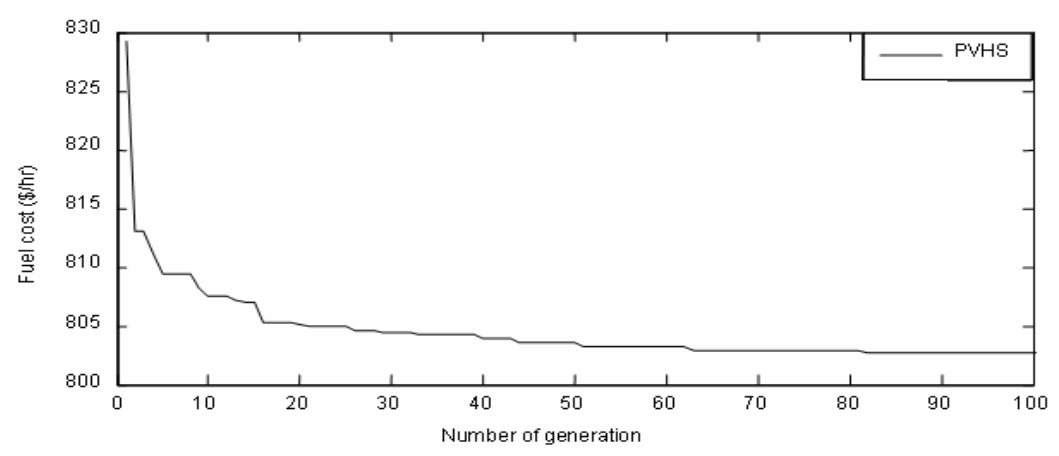

Fig. 1 Convergence of PVHS Algorithm for Case 1

\subsection{Case 2}

In this case the fuel cost characteristics of the generating units connected at bus 1 and bus 2 are having piecewise quadratic cost curve [6] to model different fuels.

$$
F_{i}\left(P_{g i}\right)=\left\{\begin{array}{c}
a_{i 1}+b_{i 1} P_{g i}+c_{i 1} P_{g i}^{2}, \quad P_{g i}^{\min } \leq P_{g i} \leq P_{g i}^{1} \\
a_{i 2}+b_{i 2} P_{g i}+c_{i 2} P_{g i}^{2}, \quad P_{g i}^{1}<P_{g i} \leq P_{g i}^{2} \\
\ldots \ldots \\
a_{i k}+b_{i k} P_{g i}+c_{i k} P_{g i}^{2}, P_{g i}^{k-1}<P_{g i} \leq P_{g i}^{\max }
\end{array}\right.
$$


where $a_{i k}, b_{i k}$ and $c_{i k}$ are the cost coefficients of the $i^{\text {th }}$ generator at the $\mathrm{k}^{\text {th }}$ interval. The other 4 generators are having same quadratic cost curve coefficients as mentioned in Case 1. The generator cost coefficients are found in [6] and the optimized parameters corresponding to minimum cost is given in Table 1 . The results of the PVHS algorithm are compared in Table 3 with other reported results using modified differential evolution algorithm (MDE) [7]. The algorithm converges quickly and the results are better than the other.

Table 3 Cost Comparison with Other Methods for Case2

\begin{tabular}{ccc}
\hline Parameter & MDE[7] & PVHS \\
\hline Best Cost & 647.846 & 647.8126 \\
Worst cost & 650.664 & 648.8110 \\
Avg cost & 648.356 & 648.2448 \\
Std cost & - & 0.2681 \\
\hline
\end{tabular}

\subsection{Case 3}

In this case the fuel cost characteristics of the generating units connected at bus 1 and bus 2 are also having a sine component to model the valve point loading effect of the generators as

$$
F_{i}\left(P_{g i}\right)=a_{i}+b_{i} P_{g i}+c_{i} P_{g i}^{2}+\left|d_{i} \sin \left(e_{i}\left(P_{g i}^{\min }-P_{g i}\right)\right)\right|
$$

where $a_{i}, b_{i}, c_{i}, \mathrm{~d}_{i}$ and $\mathrm{e}_{i}$ are the cost coefficients of the $i^{\text {th }}$ generating unit. The other 4 generators are having same quadratic cost curve coefficients as mentioned in Case 1. The generator cost coefficients are found in [6] and the optimized parameters corresponding to minimum cost is given in Table 1. The results of the PVHS algorithm are compared in Table 4 with other reported results using improved evolutionary programming (IEP) [6], and modified differential evolution algorithm (MDE) [7]. The algorithm converges quickly and the results are better than the others.

Table 4 Cost Comparison with Other Methods for Case 3

\begin{tabular}{cccc}
\hline Parameter & IEP[6] & MDE[7] & PVHS \\
\hline Best Cost & 953.573 & 930.793 & 930.7237 \\
Worst cost 958.263 & 954.073 & 930.7764 \\
Avg cost & 956.460 & 942.501 & 930.7380 \\
Std cost & 1.720 & - & 0.0162 \\
\hline
\end{tabular}

\section{Summary and Conclusions}

In this chapter, detailed discussion is carried out about the application of population variance harmony search algorithm to solve the optimal power flow problem 
in the presence of security constraints. The algorithm explores the search space quickly with the help of population variance parameter. The PVHS algorithm was tested with IEEE 30 bus test system having three different types of cost characteristics. The comparison of obtained results with other previously reported results shows the effectiveness of the algorithm.

\section{References}

1. Wood, A.J., Wollenberg, B.F.: Power Generation, Operation and Control. John Wiley \& Sons, New York (1984)

2. Momoh, J.A., El-Hawary, M.E., Adapa, R.: A review of selected optimal power flow literature to 1993 Part I: Nonlinear and quadratic programming approaches. IEEE Trans. on Power Systems 14, 96-104 (1999)

3. Momoh, J.A., El-Hawary, M.E., Adapa, R.: A review of selected optimal power flow literature to 1993 Part II: Newton, linear programming and interior point methods. IEEE Trans. on Power Systems 14, 105-111 (1999)

4. Yuryevich, J., Wong, K.P.: Evolutionary programming based optimal power flow algorithm. IEEE Trans. on Power Systems 14, 1245-1250 (1999)

5. Abido, M.A.: Optimal power flow using tabu search algorithm. Electric Power Components and Systems 30, 469-483 (2002)

6. Ongsakul, W., Tantimaporn, T.: Optimal power flow by improved evolutionary programming. Electric Power Components and Systems 34, 79-95 (2006)

7. sayah, S., Zehar, K.: Modified differential evolution algorithm for optimal power flow with non-smooth cost functions. Energy Conversion and Management 49, 3036-3042 (2008)

8. Alsac, O., Stott, B.: Optimal load flow with steady-state security. IEEE Trans. on Power Apparatus Systems 93, 745-751 (1974)

9. Abido, M.A.: Optimal power flow using particle swarm optimization. Electric Power Energy Systems 24, 563-571 (2002)

10. Bakirtzis, A.G., Biskas, P.N., Zoumas, C.E., Petridis, V.: Optimal power flow by enhanced genetic algorithm. IEEE Trans. on Power Systems 17, 229-236 (2002)

11. Roa-Sepulveda, C.A., Pavez-Lazo, B.J.: A solution to the optimal power flow using simulated annealing. Electric Power Energy Systems 25, 47-57 (2003)

12. Matlab (2007), http: / /www . mathworks . com

13. Geem, Z.W., Kim, J.H., Loganathan, G.V.: A new heuristic optimization algorithm: harmony search. Simulation 76, 60-68 (2001)

14. Geem, Z.W., Kim, J.H., Loganathan, G.V.: Harmony search optimization: application to pipe network design. Int. J. Model. Simulation 22, 125-133 (2002)

15. Lee, K.S., Geem, Z.W.: A new structural optimization method based on the harmony search algorithm. Comput. Struct. 82, 781-798 (2004)

16. Geem, Z.W., Lee, K.S., Park, Y.: Application of Harmony Search to Vehicle Routing. American Journal of Applied Sciences 2, 1552-1557 (2005)

17. Geem, Z.W.: Optimal Cost Design of Water Distribution Networks Using Harmony Search. Engineering Optimization 38, 259-280 (2006)

18. Vasebi, A., Fesanghary, M., Bathaeea, S.M.T.: Combined heat and power economic dispatch by Harmony Search Algorithm. International Journal of Electrical Power and Energy Systems 29, 713-719 (2007) 
19. Geem, Z.W.: Optimal Scheduling of Multiple Dam System Using Harmony Search Algorithm. In: Sandoval, F., Prieto, A.G., Cabestany, J., Graña, M. (eds.) IWANN 2007. LNCS, vol. 4507, pp. 316-323. Springer, Heidelberg (2007)

20. Mahdavi, M., Fesanghary, M., Damangir, E.: An improved Harmony Search Algorithm for Solving Optimization Problems. Applied Mathematics and Computation 188, 1567-1579 (2007)

21. Mukhopadhyay, A., Roy, A., Das, S., Das, S., Abraham, A.: Population-Variance and Explorative Power of Harmony Search: An Analysis. In: Third IEEE International Conference on Digital Information Management (ICDIM 2008), pp. 13-16 (2008)

22. Geem, Z.W.: Novel Derivative of Harmony Search Algorithm for Discrete Design Variables. Applied Mathematics and Computation 199, 223-230 (2008)

23. Geem, Z.W.: Harmony Search Optimization to the Pump-Included Water Distribution Network Design. Civil Engineering and Environmental Systems 26, 211-221 (2009)

24. Geem, Z.W.: Particle-Swarm Harmony Search for Water Network Design. Engineering Optimization 41, 297-311 (2009) 